\title{
Predicting the Impact of Adverse Events and Treatment Duration on Medical Resource Utilization-Related Costs in Hepatitis C Genotype 1 Treatment-Naïve Patients Receiving Antiviral Therapy
}

\author{
Essè Ifèbi Hervé Akpo $\cdot$ Karin Cerri • \\ Joris Kleintjens
}

Published online: 11 January 2015

(c) The Author(s) 2015. This article is published with open access at Springerlink.com

\begin{abstract}
Objectives Studies on medical resource utilization (MRU) and related costs are important for evaluating the potential patient management and cost-effectiveness implications of antiviral treatments for hepatitis $\mathrm{C}$ virus (HCV) infection. The objectives of this study were (i) to compare the MRU and related costs for two treatment approaches; (ii) to identify the main drivers of resource use and costs; and (iii) to assess the effects of various treatment regimen attributes on MRU-related costs in a UK clinical setting.

Methods The analysis used data collected alongside the simeprevir (SMV) phase III trials for treatment-naïve genotype $1 \mathrm{HCV}$-infected patients; these data covered outpatient consultations with specialists, emergency room visits and hospital admissions. Logistic regressions were constructed to estimate the predictors of resource utilization, and a two-part multivariable analysis model was used to determine the total costs of treatment in the UK.

Results Data on 731 patients receiving SMV plus pegylated interferon and ribavirin (SMV/PegIFN/R) or PegIFN/R were included in the analysis. While MRU was similar between the SMV and PegIFN/R groups, MRU-related costs were significantly lower in the SMV group than in the PegIFN/R group
\end{abstract}

Electronic supplementary material The online version of this article (doi:10.1007/s40273-014-0249-4) contains supplementary material, which is available to authorized users.

\section{E. I. H. Akpo $(\bowtie) \cdot J$ J. Kleintjens}

Deloitte, Diegem, Belgium

e-mail: eakpo@deloitte.com

\section{K. Cerri}

Janssen Pharmaceutica NV, Beerse, Belgium

K. Cerri

Personal Social Services Research Unit, London School of

Economics and Political Science, London, UK
$(P<0.05)$. High body mass index $(P<0.05)$, severe fibrosis $(P<0.05)$, shortened treatment duration to 24 weeks $(P<0.05)$, and anaemia and rash during treatment $(P<0.001)$ were identified as predictors of hospitalization and outpatient visits and as drivers of total costs. Univariate sensitivity analyses suggested that shortened treatment duration and lower occurrence of rash lead to large cost savings. Conclusion This study identified both baseline and ontreatment antiviral therapy characteristics as drivers of MRU-related costs for HCV patients following antiviral therapy. The shortened treatment duration and reduction in rash due to treatment with SMV triple therapy lead to substantial non-drug cost savings, compared with PegIFN/ $\mathrm{R}$ treatment. This suggests that there are potential patient management and cost-effectiveness implications associated with the choice of specific antiviral treatments.

\section{Key Points for Decision Makers}

High body mass index, severe fibrosis, shortened treatment duration of pegylated interferon to 24 weeks, and anaemia and rash during treatment were identified as drivers of medical resource utilization-related costs of genotype 1 hepatitis $\mathrm{C}$ virus infection in treatment-naïve patients.

The shortened treatment duration of pegylated interferon and reduction in rash due to simeprevir treatment lead to important non-drug cost savings, compared with pegylated interferon and ribavirin treatment.

The study suggests there are potential patient management and cost-effectiveness implications associated with the choice of specific antiviral treatments. 


\section{Introduction}

An estimated 9 million people are chronically infected with hepatitis $\mathrm{C}$ virus (HCV) in Europe [1]. Of these, approximately 215,000 are in the UK [2]. Individuals with chronic hepatitis $\mathrm{C}(\mathrm{CHC})$ often present with non-specific symptoms and may experience severe long-term complications, including cirrhosis, liver cancer and liver failure. As a result, $\mathrm{CHC}$ has been associated with high economic costs for the patient, the healthcare system and society as a whole [3]. Patruni and Nolte [4] forecasted the prevalence of HCV infection to rise from $0.44 \%$ in 2010 to $0.61 \%$ in 2035 in the UK. The related healthcare costs were predicted to rise from $£ 82.7$ million in 2012 to $£ 115$ million in 2035. Backx et al. [5] further estimated a 13-fold increase in costs (roughly $£ 2,300$ ) for patients with hepatitis $\mathrm{C}$ who failed to achieve a sustained virological response (SVR; an indication of cure) over a 5-year post-treatment period, compared with those who did not achieve SVR. Achievement of SVR is therefore a cornerstone in the management of HCV and has implications for medical service usage and costs.

For the treatment of patients with $\mathrm{CHC}$ genotype 1 infection, in several countries the standard of care is a triple regimen containing the protease inhibitors telaprevir or boceprevir in combination with pegylated interferon and ribavirin (PegIFN/R). Triple regimens with telaprevir or boceprevir yield a significantly higher SVR rate than treatment with PegIFN/R alone; however, these therapies are also associated with increased rates and severity of adverse events, including anaemia and rash, compared with PegIFN/R administered alone [6]. These adverse events sometimes require premature discontinuation of treatment and additional monitoring and management of the adverse events, compared with PegIFN/R treatment alone. These additional interventions include red blood cell transfusions and use of erythropoiesis-stimulating agents, plus close monitoring for skin manifestations, resulting in frequent visits to healthcare providers and adding to the complexity and costs (direct and indirect) of treatment. These treatment complications emphasize the need for therapeutic alternatives with improved safety profiles.

Simeprevir (SMV) is a one-pill, once-daily, potent oral HCV NS3/4A protease inhibitor approved for use in the USA, Europe, Japan and Canada, in combination with other agents, including PegIFN/R, for the treatment of adult patients with CHC [7]. Two phase III, multicentre, randomized, double-blind, placebo-controlled trials were conducted to evaluate the safety and efficacy of SMV in treatment-naïve patients (trials $\mathrm{C} 208$ and C216). The results of these trials indicated that the SMV/PegIFN/R regimen is a well-tolerated and effective therapeutic alternative for $\mathrm{HCV}$-infected patients [8, 9].
Variations in medical resource utilization (MRU) and related costs by patient characteristics, clinical factors and treatment regimen have not been widely studied in $\mathrm{HCV}$ populations. To address this need for evidence, data on MRU for routine clinical services were collected alongside the clinical studies on SMV. Hence, the primary objective of this study was to compare the MRU and related costs for patients in the SMV/PegIFN/R triple therapy arm with those in the PegIFN/R dual therapy arm. The secondary objective was to identify the main drivers of resource use and costs. The last objective was to assess the effect of various treatment regimen attributes on MRU-related costs in a UK clinical setting, with a particular focus on treatment duration and adverse events.

\section{Materials and Methodology}

\subsection{Randomized, Controlled Trial Design}

The two pivotal phase III trials (C208 and C216) were designed to investigate SMV $150 \mathrm{mg}$ once-daily dosing for 12 weeks in combination with PegIFN/R for 24 or 48 weeks (on the basis of the treatment response), compared with placebo plus PegIFN/R. Patients were assigned 2:1 to the SMV plus PegIFN/R arm or the placebo plus PegIFN/R arm, respectively. For the analyses presented in this paper, the $\mathrm{C} 208$ and C216 study data on treatmentnaïve patients were pooled to increase the sample size and allow for a more meaningful comparison. Pooling was considered appropriate, given the comparable trial designs and trial populations. The primary endpoint of the phase III studies was SVR, defined as undetectable HCV RNA at the end of treatment and HCV RNA $<25 \mathrm{IU} / \mathrm{mL}$ at 12 weeks after treatment. MRU data were collected as an exploratory endpoint in these phase III trials.

\subsection{Resource Utilization and Costs}

As part of the SMV phase III trial programme, data on MRU over and above standard protocol-driven study visits were collected, including healthcare provider visits, home visits, emergency room visits, hospitalization, and admission to an intensive care unit. Healthcare providers included social workers, gastroenterologists, hepatologists, nurses, primary care physicians, infectious disease specialists, psychiatrists, psychologists and surgeons. The MRU data were collected at each study visit. To focus on how resource utilization and related costs correlate with patient characteristics and treatment features, the costs of SMV and PegIFN/R were not included in this assessment. Protocol-mandated procedures, tests and encounters were excluded from the MRU data. 
The analysis was carried out from the perspective of the UK National Health Service (NHS). Unit costs for each resource were derived from NHS reference costs [10] or the 'Unit Costs of Health and Social Care' from the Personal Social Services Research Unit (PSSRU) [11, 12]. Costs were inflated to 2013 values, using the medical component of the Consumer Price Index [13]. The unit costs are summarized in Table 1. The total MRU-related cost per patient was calculated by multiplying each resource by its unit costs and then summing the individual MRU-related costs for each patient or treatment arm.

\subsection{Statistical Analyses}

\subsubsection{Dataset}

All analyses were performed on the basis of a subset of patients with no missing data. Extreme cost values (outliers) were defined as values with a studentized residual [a raw residual that is divided by its estimated standard deviation (SD)] greater than 2. Studentized residuals were calculated through linear regression on the total costs while controlling for variables such as age, baseline body mass index (BMI) and baseline HCV viral load. Once the patients with outlier values were removed from the dataset, descriptive statistics were used to determine MRU and to make a comparison between the two treatment arms.

\subsubsection{Resource Utilization and Costs}

To address the first objective of this study, bivariate descriptive statistics compared MRU between patients assigned to the SMV triple therapy arm and those assigned to the control group. The analyses used the chi-squared test for categorical variables and the Wilcoxon rank-sum test for the costs to determine the significance of the difference between the treatment arms at the $5 \%$ level. In addition, the equality of the median of the number of visits to specialists (a non-normally distributed variable) between the two treatment arms was evaluated with the Wilcoxon ranksum test. Subgroup analyses were also performed to determine whether patients respond differently to treatment according to their clinical factors. For this purpose, a Kruskal-Wallis test and a Wilcoxon rank-sum test were performed. More specifically, these tests investigated whether total MRU-related costs differ by interleukin-28B (IL28B) status and by fibrosis METAVIR score of F0-F2 and F3-F4, respectively.

To explore the predictors of resource utilization, the second objective of this study, stepwise binary logistic regression models, were constructed. These models specifically determined the odds of using a specific type of medical resource as a function of the following variables: patient demographics (age, gender, baseline BMI, geographical region), clinical factors (baseline HCV viral load, advanced fibrosis METAVIR scores and IL28B genotype), and treatment features (treatment status, SVR achievement, treatment duration and adverse events) (see Online Supplementary Appendix 1) [14]. The adverse events included anaemia, pruritus, rash and combinations of these events.

Besides identifying predictors of MRU, attention was also paid to predictive factors associated with adverse events. This secondary research question was pertinent in light of the increased rate of anaemia in patients treated with telaprevir and boceprevir, compared with those receiving PegIFN/R [15-17]. Gaining knowledge of the
Table 1 Unit cost per medical resource (UK tariff)

Tariff data were taken from Department of Health reference costs 2011-2012 [10], unless stated otherwise

a Tariff data were taken from from Curtis [11]

b Tariff data were taken from from Curtis [12]

c A conservative estimate of the unit costs of physiotherapy was applied because the majority of 'other visits' were related to physiotherapy and the other types of visits in this category were typically more expensive

\begin{tabular}{|c|c|c|c|c|}
\hline & \multicolumn{2}{|c|}{ Unit cost $[£], 2011-2012$} & \multicolumn{2}{|c|}{ Inflation correction to 2013 [£] } \\
\hline & $\begin{array}{l}\text { First } \\
\text { attendance }\end{array}$ & $\begin{array}{l}\text { Follow-up } \\
\text { attendance }\end{array}$ & $\begin{array}{l}\text { First } \\
\text { attendance }\end{array}$ & $\begin{array}{l}\text { Follow-up } \\
\text { attendance }\end{array}$ \\
\hline Emergency room visits & 122 & 33 & 128 & 35 \\
\hline Gastroenterologist visits & 164 & 45 & 172 & 47 \\
\hline Hepatologist visits & 216 & 27 & 227 & 28 \\
\hline Infectious disease specialist visits & 280 & 164 & 294 & 172 \\
\hline Psychiatrist visits & 178 & 178 & 187 & 187 \\
\hline Psychologist visits & 56 & 157 & 59 & 165 \\
\hline Surgeon visits & 145 & 112 & 152 & 118 \\
\hline Home visits & 70 & 29 & 74 & 31 \\
\hline Nurse visits & 21 & 21 & 22 & 22 \\
\hline Nights in intensive care units & 868 & 868 & 913 & 913 \\
\hline Primary care physician visits ${ }^{\mathrm{a}}$ & 54 & 54 & 62 & 62 \\
\hline Social worker visits ${ }^{\mathrm{a}}$ & 30 & 30 & 34 & 34 \\
\hline Nights in hospital $^{\mathrm{b}}$ & 235 & 235 & 278 & 278 \\
\hline Other visits ${ }^{\mathrm{c}}$ & 43 & - & 45 & - \\
\hline
\end{tabular}


patient characteristics and treatment factors associated with an increased risk of adverse events (when treating with dual or triple therapy) would provide clinicians with the opportunity to evaluate treatment adequacy and adherence. To tackle this point, a stepwise multinomial regression was fitted to the variable 'adverse events' while controlling for patient demographics and clinical characteristics, as well as treatment features. A series of chi-squared and Fisher exact tests were also performed to compare adverse eventdependent MRU between the two treatment arms. The Wilcoxon rank-sum test compared the median total MRUrelated costs associated with the adverse events between SMV/PegIFN/R and PegIFN/R.

The predictors of the total costs associated with the resource use were also evaluated as part of the second objective of this research. A two-part multivariable model was developed to reduce the set of patient and treatment variables to those independently associated with costs. The first part of this model was a logistic regression model predicting the probability of having any cost (e.g. having $\geq 1$ hospitalization). The second part was a generalized linear model with a log link function and gamma distribution (for skewness adjustment) to determine the amount of costs (when costs were incurred). The total MRU-related costs per designated population was then the probability of having any cost, multiplied by the predicted amount of costs. The regression analyses were performed by controlling for all factors, except for the MRU-related costs.

The performance of the developed regression models was evaluated with the Akaike information criterion (AIC) and the chi-squared test, assessing the significance of the deviation of the model from the null model, where only the intercept is captured. Multicollinearity of the regression models was further assessed using the variation inflation factor (VIF) metric. Multicollinearity was found if the VIF was greater than 2, which indicates that some variables might be redundant [18]. In general, a VIF greater than 10 indicates significant multicollinearity and unstable regression coefficients.

Finally, a two-step approach was adopted to address the third objective of the study, which was to assess the effect of various treatment characteristics on total MRU-related costs. The first step consisted of implementing a Monte Carlo simulation to establish the robustness of the predicted total MRU-related costs. The correlation models derived from the two-part multivariable analysis were fitted to the distributions obtained by Monte Carlo simulations. The Monte Carlo simulations were run for 1,000 iterations. The Cholesky decomposition method was applied to preserve the underlying correlation between the variables.

In the second step, a one-way sensitivity analysis was conducted using a range of values, which was defined as the reference case estimates $\pm 30 \%$. The reference case corresponds to the trial's population characteristics, as summarized in Online Supplementary Table S1. The tested variables included patient demographics (age, gender and BMI), clinical factors (the METAVIR score) and treatment features (adverse events and treatment duration). For each tested variable, changes in values of the reference case were applied only to the SMV triple therapy arm, to determine the potential cost savings of SMV triple therapy versus PegIFN/R dual therapy. The incremental median total MRU-related savings were then computed, and the difference between the SMV arm and the reference case was calculated. Results were plotted in a tornado diagram based on the impact of the variables on the median total MRU-related cost savings per population of 5,000 patients. All statistical analyses were carried out using $\mathrm{R}$ statistical software (http://cran.r-project.org/).

\section{Results}

\subsection{Dataset}

The initial intent-to-treat (ITT) dataset included 394 and 391 treatment-naïve patients from studies C208 and C216, respectively. From these studies, 16 patients with outliers and 38 patients with missing data were removed, leading to a consolidated dataset of 731 patients (480 SMV triple therapy-treated patients and $251 \mathrm{PegIFN} / \mathrm{R}$ dual therapytreated patients). This is equal to $93 \%$ of the ITT population.

Missing data were cases who discontinued the trial with no adverse events consequently being reported. Reasons for discontinuation in these particular cases were related to non-compliance, loss to follow-up or patients who met a virological stopping rule at week 4 . In addition, patients with a METAVIR score classified as 'other' or not reported were considered as cases with missing data. The impact of outliers on the predictors of total MRU-related costs was evaluated prior to their removal (Online Supplementary Appendix 2).

\subsection{Resource Utilization and Costs-SMV/PegIFN/R vs. PegIFN/R Groups}

Table 2 shows the proportion of patients making use of each type of medical resource, as well as the average number of visits. The results indicate that medical resource use is similar between the SMV/PegIFN/R and PegIFN/R treatment arms. Table 3 shows the total costs associated with each healthcare resource as observed in the clinical trials. The results indicate that the difference in median total MRU-related costs between treatment arms was statistically significant $(P<0.05)$. The median 
Table 2 Comparison of proportions of patients with resource utilization between simeprevir plus pegylated interferon and ribavirin (SMV/PegIFN/R) and PegIFN/R treatment

\begin{tabular}{|c|c|c|c|}
\hline Medical resource use & $\begin{array}{l}\text { SMV/PegIFN/R } \\
(N=480)\end{array}$ & $\begin{array}{l}\text { PegIFN/R } \\
(N=251)\end{array}$ & $P$ value $^{\mathrm{a}}$ \\
\hline \multicolumn{4}{|l|}{ Emergency room } \\
\hline Yes & $53(11)$ & $35(14)$ & 0.252 \\
\hline No & $427(89)$ & $216(86)$ & \\
\hline 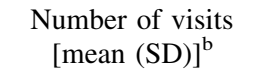 & $0.146(0.483)$ & $0.207(0.661)$ & 0.235 \\
\hline \multicolumn{4}{|l|}{ Primary care physician } \\
\hline Yes & $191(40)$ & $108(43)$ & 0.398 \\
\hline No & $289(60)$ & $143(57)$ & \\
\hline 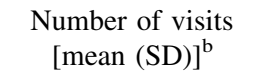 & $1.233(2.438)$ & $1.633(2.883)$ & 0.132 \\
\hline \multicolumn{4}{|l|}{ Gastroenterologist } \\
\hline Yes & $28(6)$ & $15(6)$ & 0.938 \\
\hline No & $452(94)$ & $236(94)$ & \\
\hline $\begin{array}{l}\text { Number of visits } \\
{[\text { mean (SD) }]^{\mathrm{b}}}\end{array}$ & $0.077(0.388)$ & $0.151(0.770)$ & 0.880 \\
\hline \multicolumn{4}{|l|}{ Hepatologist } \\
\hline Yes & $21(4)$ & $16(6)$ & 0.242 \\
\hline No & $459(96)$ & $235(94)$ & \\
\hline $\begin{array}{l}\text { Number of visits } \\
{[\text { mean (SD) }]^{\mathrm{b}}}\end{array}$ & $0.058(0.331)$ & $0.092(0.404)$ & 0.235 \\
\hline \multicolumn{4}{|l|}{ Nurse } \\
\hline Yes & $24(5)$ & $10(4)$ & 0.536 \\
\hline No & $456(95)$ & $241(96)$ & \\
\hline $\begin{array}{l}\text { Number of visits } \\
\text { [mean (SD) }^{\mathrm{b}}\end{array}$ & $0.177(1.219)$ & $0.104(0.818)$ & 0.525 \\
\hline \multicolumn{4}{|c|}{ Infectious disease specialist } \\
\hline Yes & $11(2)$ & $4(2)$ & 0.596 \\
\hline No & $469(98)$ & $247(98)$ & \\
\hline $\begin{array}{l}\text { Number of visits } \\
\text { [mean (SD) }^{\mathrm{b}}\end{array}$ & $0.033(0.280)$ & $0.020(0.166)$ & 0.529 \\
\hline \multicolumn{4}{|l|}{ Psychiatrist } \\
\hline Yes & $31(7)$ & $19(8)$ & 0.572 \\
\hline No & $449(93)$ & $232(92)$ & \\
\hline $\begin{array}{l}\text { Number of visits } \\
{[\text { mean (SD) }]^{\mathrm{b}}}\end{array}$ & $0.188(1.000)$ & $0.195(0.983)$ & 0.584 \\
\hline \multicolumn{4}{|l|}{ Psychologist } \\
\hline Yes & $12(3)$ & $11(4)$ & 0.166 \\
\hline No & $468(97)$ & $240(96)$ & \\
\hline 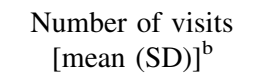 & $0.129(1.270)$ & $0.147(0.954)$ & 0.175 \\
\hline \multicolumn{4}{|l|}{ Surgeon } \\
\hline Yes & $29(6)$ & $23(9)$ & 0.119 \\
\hline No & $451(94)$ & $228(91)$ & \\
\hline 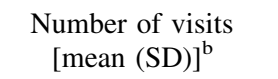 & $0.090(0.406)$ & $0.171(0.650)$ & 0.109 \\
\hline \multicolumn{4}{|c|}{ Inpatient (hospitalization) } \\
\hline Yes & $23(5)$ & $21(8)$ & 0.054 \\
\hline No & $457(95)$ & $230(92)$ & \\
\hline
\end{tabular}

Table 2 continued

\begin{tabular}{|c|c|c|c|}
\hline Medical resource use & $\begin{array}{l}\text { SMV/PegIFN/R } \\
(N=480)\end{array}$ & $\begin{array}{l}\text { PegIFN/R } \\
(N=251)\end{array}$ & $P$ value $^{\mathrm{a}}$ \\
\hline 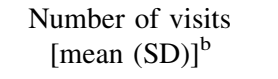 & $0.271(1.669)$ & $0.446(1.899)$ & 0.052 \\
\hline \multicolumn{4}{|l|}{ Home visits } \\
\hline Yes & $5(1)$ & $4(2)$ & - \\
\hline No & $475(99)$ & $247(98)$ & \\
\hline $\begin{array}{l}\text { Number of visits } \\
{[\text { mean (SD) }]^{\mathrm{b}}}\end{array}$ & $0.015(0.164)$ & $0.032(0.333)$ & - \\
\hline \multicolumn{4}{|l|}{ Social workers } \\
\hline Yes & $7(2)$ & $2(1)$ & - \\
\hline No & $473(98)$ & $249(99)$ & \\
\hline 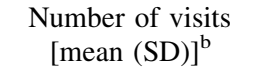 & $0.083(0.765)$ & $0.016(0.199)$ & - \\
\hline \multicolumn{4}{|l|}{ Intensive care unit } \\
\hline Yes & $1(0)$ & $3(1)$ & - \\
\hline No & $479(100)$ & $248(99)$ & \\
\hline $\begin{array}{l}\text { Number of visits } \\
{[\text { mean }(\mathrm{SD})]^{\mathrm{b}}}\end{array}$ & $0.008(0.183)$ & $0.028(0.288)$ & - \\
\hline \multicolumn{4}{|l|}{ Other visits } \\
\hline Yes & 177 (37) & $97(39)$ & 0.639 \\
\hline No & $303(63)$ & $154(61)$ & \\
\hline $\begin{array}{l}\text { Number of visits } \\
{[\text { mean }(\mathrm{SD})]^{\mathrm{b}}}\end{array}$ & $0.360(0.481)$ & $0.370(0.484)$ & 0.639 \\
\hline
\end{tabular}

The data are expressed as $n(\%)$, unless stated otherwise $S D$ standard deviation

${ }^{a} P$ values were not computed for variables with fewer than 10 data points in the two groups, to avoid statistical errors and inconclusive results. The chi-squared test was used to test for equality of proportions between the two treatment arms $(\alpha=5 \%)$. Of note, the Fisher exact test was used when the sample size was less than 10 in one treatment group but greater than 10 in the other (e.g. infectious disease specialist visits)

b Though the hypothesis of equality of the median number of visits between the two treatment arms was evaluated with the Wilcoxon rank-sum test $(\alpha=5 \%)$, the mean (SD) values are displayed in the current table as the median values were consistently equal to zero

total MRU-related costs per patient were less in the SMV triple therapy arm [median £62; interquartile range (IQR) 293] than in the PegIFN/R arm (median £124; IQR 426). The mean $( \pm \mathrm{SD})$ total MRU-related costs were $£ 296 \pm 675$ and $£ 409 \pm 881$ in the SMV/PegIFN/R arm and the PegIFN/R, respectively.

The subgroup analysis shows that patients with advanced fibrosis METAVIR F3-F4 status had greater MRU-related expenditures, compared with patients with mild/moderate liver fibrosis (METAVIR F0-F2; $P<0.001$ ) (Table 4). IL28B gene polymorphisms are well recognized as being closely associated with outcomes of PegIFN/R therapy. The Kruskal-Wallis test shows that the total MRU-related cost was similar between the three IL28B genotypes (CC, CT and TT) (Table 4). 
Table 3 Comparison of the median and mean medical resource utilization (MRU)-related costs per patient between simeprevir plus pegylated interferon and ribavirin (SMV/PegIFN/R) and PegIFN/R treatment

\begin{tabular}{|c|c|c|c|c|c|c|c|c|c|}
\hline & \multicolumn{4}{|c|}{ SMV/PegIFN/R costs $[£]$} & \multicolumn{4}{|c|}{ PegIFN/R costs $[£]$} & \multirow[t]{2}{*}{$P$ value } \\
\hline & Median & Mean & $\mathrm{IQR}$ & $n^{\mathrm{a}}$ & Median & Mean & IQR & $n^{\mathrm{a}}$ & \\
\hline Emergency room & 0 & 15 & - & 53 & 0 & 20 & - & 35 & 0.235 \\
\hline Primary care physician & 0 & 77 & - & 191 & 0 & 101 & 124 & 108 & 0.132 \\
\hline Gastroenterologist & 0 & 11 & - & 28 & 0 & 15 & - & 15 & 0.880 \\
\hline Hepatologist & 0 & 10 & - & 21 & 0 & 15 & - & 16 & 0.235 \\
\hline Nurse & 0 & 4 & - & 24 & 0 & 2 & - & 10 & 0.525 \\
\hline Infectious disease specialist & 0 & 9 & - & 11 & 0 & 5 & - & 4 & 0.529 \\
\hline Psychiatrist & 0 & 35 & - & 31 & 0 & 37 & - & 19 & 0.584 \\
\hline Psychologist & 0 & 19 & - & 12 & 0 & 20 & - & 11 & 0.175 \\
\hline Surgeon & 0 & 13 & - & 29 & 0 & 23 & - & 23 & 0.109 \\
\hline Inpatient (hospitalization) & 0 & 75 & - & 23 & 0 & 124 & - & 21 & 0.052 \\
\hline Home visits & 0 & 1 & - & 5 & 0 & 2 & - & 4 & NA \\
\hline Social workers & 0 & 3 & - & 7 & 0 & 1 & - & 2 & NA \\
\hline Intensive care unit & 0 & 8 & - & 1 & 0 & 25 & - & 3 & NA \\
\hline Total costs for all patients & 62 & $296($ SD 675) & 293 & $480^{\mathrm{b}}$ & 124 & $409(\mathrm{SD} 881)$ & 426 & $251^{\mathrm{b}}$ & $<0.05$ \\
\hline Total costs with MRU & 186 & 454 (SD 792) & 409 & $313^{\mathrm{b}}$ & 293 & $593(\mathrm{SD} 1,010)$ & 528 & $173^{\mathrm{b}}$ & $<0.05$ \\
\hline
\end{tabular}

The median and average reported MRU-related costs, as well as the IQRs, correspond to the costs across the whole studied population (including patients with no cost). Except for patients visiting primary care physicians in the PegIFN/R arm, the IQRs associated with all other medical resources were equal to 0 , meaning that the majority of the patients do not have a cost for the corresponding medical service. The Wilcoxon ranksum test was used to test the hypothesis that the median MRU-related costs between the treatment arms is similar $(\alpha=5 \%)$. The Wilcoxon rank-sum test was not computed for medical resources with fewer than 10 data points, to avoid statistical errors and inconclusive results

$I Q R$ interquartile range, $N A$ not applicable, $S D$ standard deviation

${ }^{a}$ Indicates the number of patients having a non-null cost for a specific medical service

${ }^{b}$ Full size of the selected population

\subsection{Predictors of Resource Utilization and Costs}

To evaluate the predictors of resource utilization, the odds ratios (ORs) for the use of specific medical resources were calculated. Table 5 summarizes the outputs of the validated stepwise logistic regression in which the selected set of variables were included as predictors of resource utilization. Hospitalization [OR 2.251; $95 \%$ confidence interval (CI) 1.207-4.198] and visits to hepatologists (OR 2.401; $95 \%$ CI 1.192-4.836) generally increased with advanced fibrosis METAVIR F3-F4 stages $(P<0.05)$. On the other hand, the odds of hospitalization (OR $0.528 ; 95 \%$ CI $0.285-0.978$ ) and visits to hepatologists (OR 0.448; $95 \%$ CI 0.224-0.898) were reduced when treatment duration was shortened to 24 weeks $(P<0.05)$ and SVR by week 12 (SVR12) was achieved $(P<0.05)$. The odds of consulting the primary care physician were more than twofold higher in patients with rash (OR 2.299; $95 \%$ CI $1.370-3.860 ; P<0.01)$ and in those with both anaemia and rash (OR 2.156; $95 \%$ CI 1.005-4.624; $P<0.05$ ), compared with those with no adverse events. Moreover, patients from the Asia-Pacific region were more likely to visit primary care physicians than those from Europe (OR 0.177 ; $95 \%$ CI $0.086-0.363 ; P<0.001$ ), North America
(OR 0.399; $95 \%$ CI 0.191-0.830; $P<0.05$ ) and South America (OR 0.040; $95 \%$ CI 0.014-0.116; $P<0.001$ ). For the other type of medical resources, clinical factors and treatment features were found not to be predictors.

To predict the costs associated with treating patients with HCV infections, we adopted a two-model approach that predicts the probability of having any costs and the amount of those costs. The average total costs can then be calculated by multiplying the probability by the amount.

The logistic regression model showed that age (OR 1.045 ; $95 \%$ CI 1.029-1.061) and BMI (OR 1.032; $95 \%$ CI 1.001-1.063) significantly increased the odds of having any costs (Table 6). These results indicate that for each additional year in the patient's age, the odds of having any costs increases by approximately $5 \%$. Similarly, the significant odds ratio of 1.032 for BMI indicates that for each $1 \mathrm{~kg} / \mathrm{m}^{2}$ increase in BMI, a patient has a $3 \%$ higher chance of incurring costs. The occurrence of adverse events, especially of rash (OR 3.062; $95 \%$ CI 1.722-5.447) and the joint occurrence of anaemia and rash (OR 4.662; $95 \%$ CI 1.577-13.783) greatly increased the odds of any cost being incurred, as compared with none of the selected adverse events occurring. The results of the stepwise gamma log link regression analysis showed that factors 
Table 4 Subgroup analysis comparing total medical resource utilization (MRU)-related costs by fibrosis METAVIR stages and by interleukin-28B (IL28B) gene polymorphism

\begin{tabular}{lllll}
\hline & \multicolumn{2}{l}{ Costs $[£]$} & & \\
\cline { 2 - 3 } & Median value $^{\mathrm{b}}$ \\
& Mean & IQR $n / N^{\mathrm{a}}$ & \\
\hline
\end{tabular}

Comparison of total MRU-related costs by fibrosis METAVIR stage METAVIR stage

$\begin{array}{llllll}\text { F0-F2 } & 62 & 295 & 294 & 340 / 539 & <0.001 \\ \text { F3-F4 } & 169 & 446 & 393 & 146 / 192 & \end{array}$

Comparison of total MRU-related costs by IL28B gene polymorphism IL28B polymorphism

$\begin{array}{llllll}\text { CC } & 62 & 308 & 276 & 145 / 214 & 0.874 \\ \text { CT } & 84 & 349 & 379 & 268 / 408 & \\ \text { TT } & 62 & 336 & 340 & 73 / 109 & \end{array}$

The median and average reported MRU-related costs, as well as the IQRs, correspond to the costs across the whole studied population (including patients with no cost)

$I Q R$ interquartile range

${ }^{a}$ Indicates the number of patients having a non-null cost over the total number of patients with respect to METAVIR stage or IL28B gene polymorphism

b The Wilcoxon rank-sum test was used to test the hypothesis that the median total MRU-related costs are similar between the METAVIR stages $(\alpha=5 \%)$. The Kruskal-Wallis test was used to test the hypothesis that the median total MRU-related costs are similar across the different types of polymorphism at the IL28B locus $(\alpha=5 \%)$

with significant odds ratios were age (OR 0.982; $95 \%$ CI 0.969-0.996), male gender (OR 0.722; $95 \%$ CI 0.548-0.952) and shortened treatment duration (OR 0.731; $95 \%$ CI 0.554-0.964); these factors were associated with 2, 28 and $27 \%$ reductions in total MRU-related costs, respectively. Finally, the total MRU-related costs of patients with advanced fibrosis METAVIR stages F3-F4 were estimated to be $44 \%$ higher than those of patients with no advanced fibrosis (OR 1.438; $95 \%$ CI 1.047-1.974) (Table 6).

The validity of all models was assessed using VIF statistics. In all models, VIF values were greater than 2 but less than 10 for the variable SMV (which takes the value 'yes' if patients were on SMV triple therapy, and 'no' if not) and the variable PR24 (which takes the value 'yes' if treatment duration was shortened to 24 weeks, and 'no' if not). As an example, the VIF values for SMV and PR24 were 5.098 and 5.459, respectively, indicating that collinearity between the predictors of the odds of total MRUrelated costs exists. These results aligned with the trial observations-that is, patients in the SMV triple therapy arm achieved SVR12 compared with patients in the control arm who did not $[8,9]$. Consequently, patients in the SMV triple therapy arm were eligible for a shortened treatment duration from 48 to 24 weeks. Forward and backward stepwise regression, model selection for predictors of MRU
Table 5 Odds of medical resource utilization, by explanatory variable

\begin{tabular}{|c|c|c|c|}
\hline & OR & $95 \% \mathrm{CI}$ & $P$ value \\
\hline \multicolumn{4}{|l|}{ Hospitalization } \\
\hline Intercept & 0.068 & $(0.042-0.112)$ & $<0.001$ \\
\hline Shortened dual therapy (PR24) ${ }^{\mathrm{a}}$ & 0.528 & $(0.285-0.978)$ & $<0.05$ \\
\hline METAVIR stage $(\mathrm{F} 3-\mathrm{F} 4)^{\mathrm{a}}$ & 2.251 & $(1.207-4.198)$ & $<0.05$ \\
\hline \multicolumn{4}{|l|}{ Hepatologist } \\
\hline Intercept & 0.117 & $(0.036-0.384)$ & $<0.001$ \\
\hline METAVIR stage (F3-F4) ${ }^{\mathrm{a}}$ & 2.401 & $(1.192-4.836)$ & $<0.05$ \\
\hline SVR12 ${ }^{\mathrm{a}}$ & 0.448 & $(0.224-0.898)$ & $<0.05$ \\
\hline \multicolumn{4}{|l|}{ Primary care physician } \\
\hline Intercept & 0.234 & $(0.064-0.853)$ & $<0.05$ \\
\hline Europe $^{\mathrm{b}}$ & 0.177 & $(0.086-0.363)$ & $<0.001$ \\
\hline North America ${ }^{\mathrm{b}}$ & 0.399 & $(0.191-0.830)$ & $<0.05$ \\
\hline South America ${ }^{\mathrm{b}}$ & 0.040 & $(0.014-0.116)$ & $<0.001$ \\
\hline Age & 1.035 & $(1.018-1.051)$ & $<0.001$ \\
\hline BMI & 1.029 & $(0.998-1.060)$ & 0.070 \\
\hline Shortened dual therapy (PR24) & 0.771 & $(0.552-1.076)$ & 0.126 \\
\hline Anaemia $^{c}$ & 1.184 & $(0.686-2.045)$ & 0.545 \\
\hline Anaemia and pruritus ${ }^{c}$ & 1.764 & $(0.725-4.291)$ & 0.211 \\
\hline Anaemia and $\operatorname{rash}^{c}$ & 2.156 & $(1.005-4.624)$ & $<0.05$ \\
\hline Anaemia and rash and pruritus ${ }^{\mathrm{c}}$ & 1.445 & $(0.415-5.032)$ & 0.563 \\
\hline Pruritus $^{c}$ & 1.587 & $(0.962-2.620)$ & 0.071 \\
\hline $\operatorname{Rash}^{\mathrm{c}}$ & 2.299 & $(1.370-3.860)$ & $<0.01$ \\
\hline Rash and pruritus ${ }^{\mathrm{c}}$ & 1.847 & $(0.988-3.452)$ & 0.055 \\
\hline
\end{tabular}

Multivariable regression analyses were conducted for medical resources with more than 10 patient records (excluding therefore home visits, social worker visits and nights in intensive care units). For each type of medical resource, 'yes' (or 'no') was assigned if a patient incurred a cost (or did not incur a cost, respectively). Stepwise logistic regressions were then performed while controlling for covariates as appropriate. Only the medical resources for which clinical outcomes and treatment features determined the odds of utilization are captured in this table

$B M I$ body mass index, $C I$ confidence interval, $O R$ odds ratio, SVR12 sustained viral response by week 12

a For each of the relevant predictors, the reference group is 'no'

b For each of the relevant predictors, the reference group is AsiaPacific (including Australia and New-Zealand)

${ }^{c}$ For each of the relevant predictors, the reference group is absence of the selected adverse event(s)

and for MRU-related costs with the lowest AIC score consistently resulted in models where PR24 was selected in place of SMV.

\subsection{Predictors of Adverse Events and Associated Costs}

Table 7 reports the results of the stepwise multinomial regression used to predict the factors associated with ontreatment adverse events. The analysis was conducted for each treatment arm. To tackle the convergence issues 
Table 6 Multivariable analysis of the effect of demographics, clinical and treatment characteristics on total costs for treatment-naïve patients

\begin{tabular}{|c|c|c|c|c|}
\hline & Estimate & SE & OR $(95 \% \mathrm{CI})$ & $\mathrm{P}$ value \\
\hline \multicolumn{5}{|c|}{ Logistic regression for the probability of having any cost ${ }^{\mathrm{a}}$} \\
\hline Intercept & -2.216 & 0.511 & $0.109(0.040,0.297)$ & $<0.001$ \\
\hline Age & 0.044 & 0.008 & $1.045(1.029,1.061)$ & $<0.001$ \\
\hline Body mass index (BMI) & 0.031 & 0.015 & $1.032(1.001,1.063)$ & $<0.05$ \\
\hline Shortened dual therapy (PR24) & -0.317 & 0.172 & $0.728(0.519,1.021)$ & 0.066 \\
\hline Anaemia & 0.069 & 0.269 & $1.071(0.632,1.815)$ & 0.799 \\
\hline Anaemia and Pruritus & 1.053 & 0.578 & $2.865(0.923,8.897)$ & 0.069 \\
\hline Anaemia and Rash & 1.539 & 0.553 & $4.662(1.577,13.783)$ & $<0.01$ \\
\hline Anaemia and Rash and Pruritus & 1.090 & 0.799 & $2.975(0.621,14.245)$ & 0.172 \\
\hline Pruritus & 0.318 & 0.250 & $1.374(0.841,2.244)$ & 0.204 \\
\hline Rash & 1.119 & 0.294 & $3.062(1.722,5.447)$ & $<0.001$ \\
\hline Rash and Pruritus & 0.179 & 0.313 & $1.195(0.647,2.207)$ & 0.568 \\
\hline \multicolumn{5}{|c|}{ Log-Gamma regression for predicting the amount of $\operatorname{cost}^{\mathrm{b}}$} \\
\hline Intercept & 7.282 & 0.343 & $1453.781(742.675,2845.766)$ & $<0.001$ \\
\hline Age & -0.018 & 0.007 & $0.982(0.969,0.996)$ & $<0.01$ \\
\hline Male & -0.326 & 0.141 & $0.722(0.548,0.952)$ & $<0.05$ \\
\hline Shortened dual therapy (PR24) & -0.313 & 0.141 & $0.731(0.554,0.964)$ & $<0.05$ \\
\hline METAVIR stage (F3-F4) & 0.363 & 0.162 & $1.438(1.047,1.974)$ & $<0.05$ \\
\hline
\end{tabular}

$B M I$ body mass index, $C I$ confidence interval, $M R U$ medical resource utilization, $O R$ odds ratio, $S E$ standard error

${ }^{a}$ Describes the results of the logistic regression fitted to the variable total MRU-related costs while controlling for covariates as appropriate. The resulting equation is as follows: $\ln \left(\frac{P(x)}{1-P(x)}\right)=-2.216+0.044 \times$ Age $+0.031 \times \mathrm{BMI}-0.317 \times \mathrm{PR} 24+0.069 \times$ Anaemia $+1.053 \times$ (Anaemia and Pruritis) $+1.539 \times($ Anaemia and Rash $)+1.090 \times($ Anaemia and Rash and Pruritis $)+0.318 \times$ Pruritis $+1.119 \times$ Rash + $0.179 \times$ (Rash and Pruritus) where $P(\mathrm{x})$ is the probability of having any cost

b Shows the results of the gamma-log link regression fitted to the variable total MRU-related costs. The resulting equation is as follows: $\ln (C(x))=7.282-0.018 \times$ Age $-0.326 \times$ Male $-0.313 \times$ PR24 $+0.363 \times$ F3F4 where $C(\mathrm{x})$ defines the amount of total MRU-related costs

observed with PegIFN/R data, the levels of the categorical variable 'adverse events' were defined as follows: anaemia, pruritus, rash, multiple adverse events and absence of adverse event. The results showed that gender, age and BMI were patient demographic attributes predicting the occurrence of adverse events, irrespective of the treatment choice. With respect to gender, male patients were less likely to develop anaemia than female patients when treated with either SMV/PegIFN/R [relative risk (RR) 0.369; $95 \%$ CI $0.193-0.705]$ or PegIFN/R (RR 0.360; $95 \%$ CI $0.160-0.811)$. For every additional year of age, there was an increase in the risk of anaemia and of multiple adverse events, regardless of the medications. Each additional year in patient age increased the risks of pruritus and rash by 5 and $4 \%$, respectively, in patients treated with SMV/PegIFN/R (Table 7). Lastly, each unit increase in BMI reduced the risk of anaemia by $91 \%$ in patients treated with SMV/PegIFN/R, and reduced the risk of pruritus by about $92 \%$ (as well as reducing the risk of multiple adverse events) in patients treated with PegIFN/R. Of the treatment features included in the analysis, only SVR12 was found to be significantly associated with the risk of adverse events. However, this was specific to PegIFN/R only. Achieving SVR12 when treated with PegIFN/R was associated with significant increases of 2, 4 and $3 \%$ in the risks of anaemia, rash and multiple adverse events, respectively (Table 7). Finally, the series of chi-squared and Fisher exact tests indicated that there was insufficient evidence to relate a significant difference in adverse eventinduced MRU between the two treatment arms (see Online Supplementary Table S2). There were no differences between SMV/PegIFN/R and PegIFN/R in median total MRU-related costs for managing the adverse events. With respect to the absence of the selected adverse events, the median total MRU-related costs were significantly higher in patients treated with PegIFN/R (median £54; IQR 313) than in those receiving SMV/PegIFN/R (median £45; IQR £190) (see Online Supplementary Table S3).

\subsection{Effect of Changing Treatment Regimen Attributes on MRU-Related Cost Savings}

The Monte Carlo simulations of total MRU-related costs, using the characteristics of the reference case, resulted in predicted costs per patient in the SMV/PegIFN/R arm (median £286; IQR 74) and the PegIFN/R arm (median 


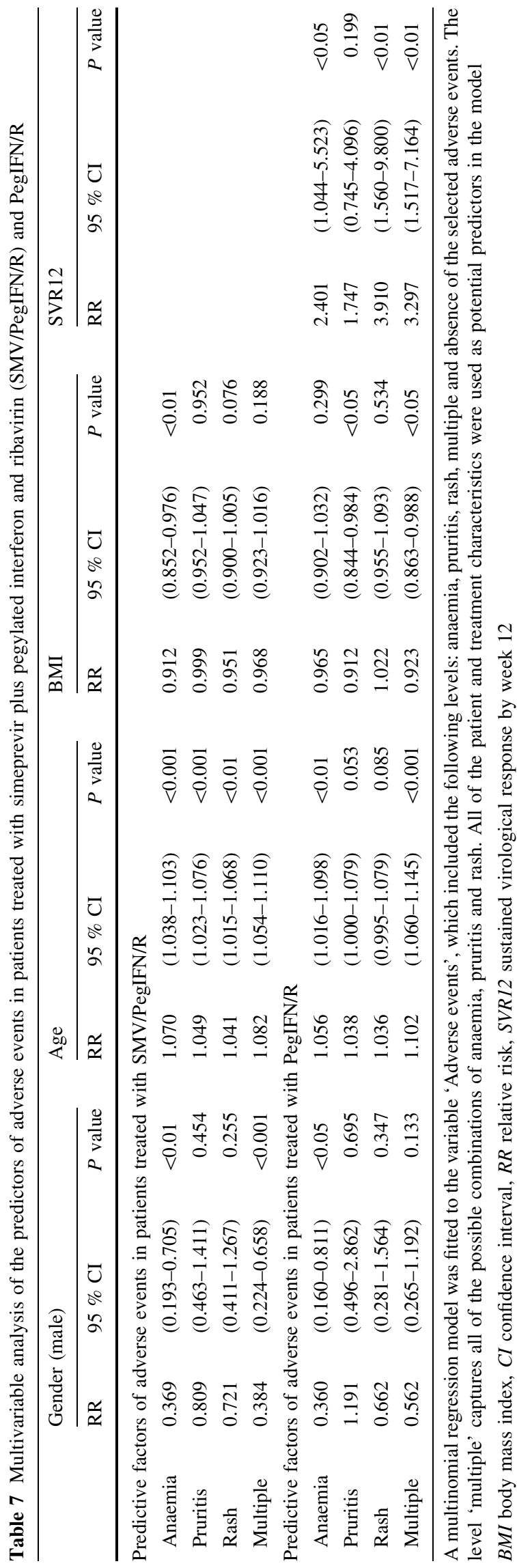

$£ 421$; IQR 108) that were similar to the costs observed in the studies (Fig. 1). The Wilcoxon rank-sum test demonstrated a difference in the median simulated MRU-related costs between the two treatment arms $(P<0.001)$. The simulated mean $( \pm \mathrm{SD})$ total MRU-related costs per patient were $£ 296 \pm 62$ and $£ 431 \pm 79$ in the SMV/PegIFN/R arm and the PegIFN/R arm, respectively.

Figure 2 shows the results from a one-way sensitivity analysis in a tornado diagram to determine by how much the incremental costs would change if the input parameter was varied by $\pm 30 \%$ of the reference case value. The median MRU-related savings were $£ 654,787$ per cohort of 5,000 patients in the reference case. The analysis shows that 'shortened dual therapy (PR24)' and 'rash' are the most important treatment-related drivers of cost savings. The joint occurrence of anaemia and rash resulted in less cost savings than the reference case cost savings. The patient characteristics 'BMI' and 'gender (male)' have the largest impact on cost savings, compared with the reference case.

\subsection{Discussion}

The results from the pivotal phase III clinical trials indicate that SMV plus PegIFN/R is a well-tolerated and effective therapeutic alternative for $\mathrm{HCV}$-infected patients. SMV/ PegIFN/R is associated with high SVR12 rates and has an adverse event profile similar to that of PegIFN/R alone. In line with these findings, studies C208 and C216, which included treatment-naïve patients, showed that SMV/PegIFN/R-treated patients had lower non-drug costs than PegIFN/R-treated patients. MRU did not differ significantly between the two treatment arms, probably because of the large heterogeneity of the resource utilization data, which capture many different types of resources.

As expected, the subgroup analysis showed that MRUrelated costs increase with the severity of liver fibrosis. These results aligned with the results of the logistic regression analysis, indicating that patients with advanced fibrosis had greater odds of medical services utilization, including hepatologist visits and hospitalization. Consequently, the total MRU-related costs are expected to increase, as demonstrated with the multivariable regression analysis.

Polymorphisms at the IL28B locus have been described as strong predictors of treatment response to PegIFN/R [19-21]. Patients who have the IL28B-CC genotype are more likely to have SVR with PegIFN/R than patients who have the CT or TT genotype. The stratified analysis of total MRU-related costs by IL28B genotype showed similar expenditures among the three classes of IL28B polymorphisms. In agreement with the regression analyses, these results demonstrated that IL28B polymorphisms are hardly 
predictors of MRU in the treatment of patients with protease inhibitors. Moreover, costs savings were unlikely to be more prominent in patients with the CC genotype than in those with the CT or TT genotypes.

In the multivariable analysis, age, gender (male) and shortened treatment duration were significantly associated with lower total MRU-related costs, whereas advanced liver fibrosis was associated with higher costs. The finding that patients with a shortened treatment duration incurred lower costs was consistent with the higher frequency of patients in the SMV arm who stopped their treatment after 24 weeks because they had achieved SVR12. Indeed, after controlling for baseline and treatment characteristics, we found that patients with a shortened treatment duration of

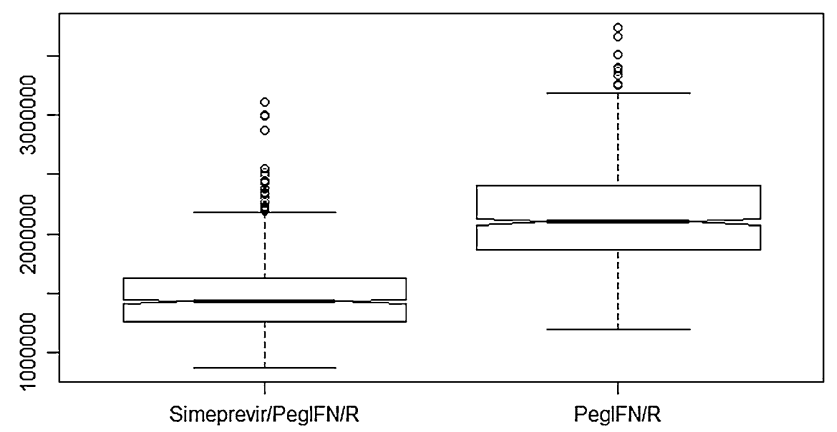

Fig. 1 Predicted medical resource utilization (MRU)-related costs [£] per cohort associated with simeprevir plus pegylated interferon and ribavirin (simeprevir/PegIFN/R) versus PegIFN/R. Notched box-plot summarizing the distribution of MRU-related costs based on the trial's population characteristics. A cohort of 5,000 patients was simulated, and 1,000 Monte Carlo simulations were performed. The absence of overlap between the notches of simeprevir/PegIFN/R and PegIFN/R indicates that the medians are significantly different from each other. The median MRU-related costs per cohort in the simeprevir/PegIFN/R arm and the PegIFN/R arm were $£ 1,461,512$ and $£ 2,124,041$, respectively
24 weeks incurred only three quarters of the costs incurred by patients treated over 48 weeks. Not surprisingly, patients with a METAVIR F3-F4 score (advanced fibrosis) had 1.5 times higher costs than patients with no advanced fibrosis. Overall, the odds of having any costs were determined significantly by age, BMI and the occurrence of rash or of both anaemia and rash. This result is confirmed by the previous findings that HCV complications are correlated with age and BMI [22-24]. A high BMI has indeed been demonstrated to be positively associated with the pathogenesis of steatosis and fibrosis and to be an independent risk factor for non-response to antiviral therapy [24-26].

These observations aligned with the results of the univariate sensitivity analysis. The tornado diagram shows that treatments leading to an increase in the proportion of patients with a shortened treatment duration, and to a reduction in the occurrence rate of rash, are associated with significant savings. Indeed, MRU-related cost savings increased by $5 \%$ when the reference case value of the variable 'shortened dual therapy (PR24)' increased by $30 \%$. On the other hand, cost savings decreased by $31 \%$ and increased by $9 \%$ when the reference case values of the variables 'shortened dual therapy (PR24)' and 'rash' were reduced by $30 \%$, respectively. The joint occurrence of anaemia and rash has a small impact on the cost savings.

On the basis of patient demographics and clinical characteristics, BMI followed by male gender and age were the most significant drivers of the savings that could be realised when treating treatment-naïve patients with SMV/ PegIFN/R compared with PegIFN/R. Higher BMI values resulted in lower cost savings, due to an increase in SMV/ PegIFN/R resource utilization. Similarly, higher rates of advanced liver fibrosis were associated with reduced cost savings. Overall, the results underscore the importance of

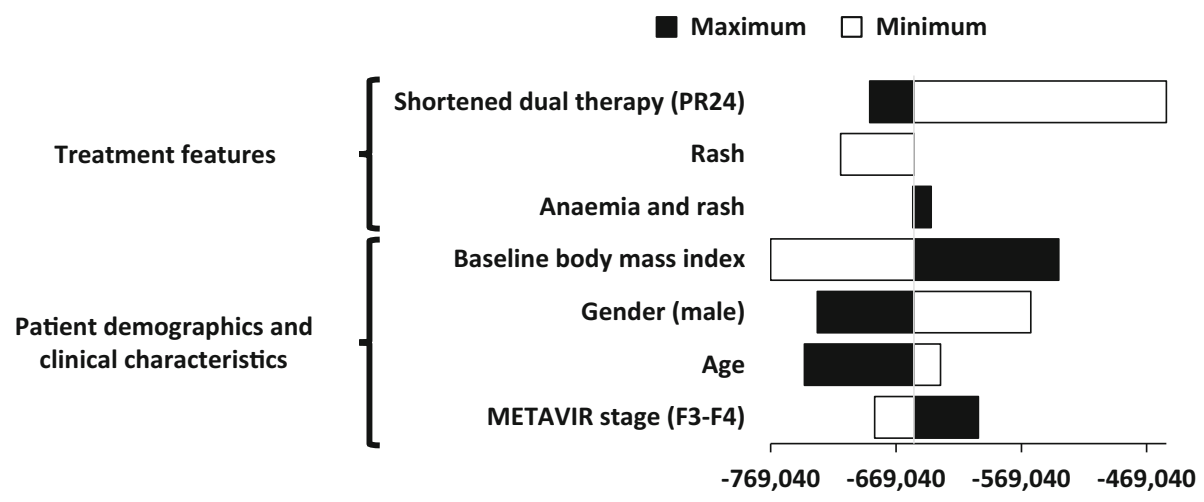

Fig. 2 Univariate sensitivity analysis of simeprevir plus pegylated interferon and ribavirin (simeprevir/PegIFN/R) on the median medical resource utilization (MRU)-related cost savings per cohort. The tornado diagram shows the degree to which uncertainty in individual variables affects the median MRU-related cost savings. Univariate sensitivity analyses were conducted by changing by $\pm 30 \%$ the values of the reference case. The changes were applied only to the simeprevir/PegIFN/R arm, and for each change in the parameter value, 1,000 Monte Carlo iterations were performed. The simulated population size was about 5,000 patients. Savings were defined as the difference between the costs in the simeprevir/PegIFN/R arm and the PegIFN/R arm 
considering the length of the treatment period and the adverse event profile when considering therapeutic agent options.

Because adverse events are the main drivers of costs in treating hepatitis $\mathrm{C}$, it is important that they are accurately incorporated into the regression model. In this study, adverse events could be defined either as standalone variables or as categorical variables. Given that each combination of adverse events was observed only in a few patients (see Online Supplementary Table S1), defining them as a standalone variable would affect the robustness of the model, because of the uncertainty associated with each combination. Therefore, the adverse events were captured in one seven-level categorical variable (anaemia, pruritus, rash and their combinations) instead. This allowed aggregation of all adverse events and their combinations together into a single variable, thereby reducing the uncertainty associated with the rarely observed adverse event combinations. In addition, this method led to the model with the best fit, based on the AIC scores as compared with models with two levels (presence/absence) and five levels (absence, anaemia, rash, pruritus and multiple) [data not shown].

This study also identified key baseline factors and treatment features predictive of adverse events in patients treated with SMV/PegIFN/R and PegIFN/R. In a real-life setting study, Singh et al. [17] reported a significant association between an increase in the odds of anaemia and female gender, as well as hypertension. The protective effect of obesity on anaemia was further indicated, as well as the correlation between increased age and a trend towards developing severe anaemia. There were no differences in the development of severe anaemia when triple and dual therapies were compared. Observations from other studies have reported that anaemic patients were more likely to be older, to be female and to have lower a BMI [27-30]. A detailed analysis of the currently selected population indicated that the average female patient's BMI was lower than that of the male patient population, potentially explaining the higher risk of anaemia in this patient population group (data not shown). Regarding age, the female patient population also tends to be older than the male patient population, further supporting the higher risk of anaemia in this subgroup. The strong association between SVR12 and anaemia in patients treated with PegIFN/R was not surprising, given the well-documented relation between experiencing anaemia and long exposure to PegIFN/R to achieve SVR $[15,31]$. This is likely explained by the substantial exposure to ribavirin [32]. Ribavirin can be used to increase the SVR rate or to reduce the duration of exposure to a treatment regimen without reducing the SVR rate [33]. Hence, the absence of a correlation between adverse events and SVR12 in patients treated with SMV/PegIFN/R supports the superiority of SMV/PegIFN/R over PegIFN/R in terms of risk-benefit [8, 9].

The analyses performed here excluded the costs of antiviral and co-medications, which typically constitute the largest part of the total treatment costs for HCV patients $[34,35]$. The reason for this was that the first aim of this study was to identify patient and treatment characteristics that would influence MRU-and thus the total cost of care-so that these could be better managed, as in other therapeutic areas, such as oncology, diabetes and human immunodeficiency virus (HIV) infection [36-38]. In addition, patients were assigned at random to each treatment regimen (as per protocol), so, by definition, there was no correlation between patient characteristics and drug regimen utilization. Last, with respect to co-medications, no differences in drug intake (defined as the treatment length multiplied by the dose frequency) and in drug consumption (defined as the treatment length multiplied by the dose frequency and the unit of the dosage form-tablet/capsule or suspension) were found between the two treatment arms (data not shown).

The MRU-related costs in our study were within the ranges of treatment costs previously published. In the German context, the costs for outpatient visits were $€ 355$ (£293), which represent about $2 \%$ of the total treatment cost for HCV patients [34, 35, 39]. Athanasakis et al. [40] estimated the annual cost per patient for patients with a METAVIR score of F0-F3 to be $€ 643$ ( $\$ 530$ ), excluding the cost of medications. Davis et al. reported the total mean $( \pm \mathrm{SD})$ medical service costs per patient with genotype 1 as being $€ 428 \pm 2,996$ ( $£ 353 \pm 2,469)$. Emergency room visits were $€ 35 \pm 106$ ( $£ 29 \pm 87)$, and general office visits were $€ 111 \pm 225$ (£91 \pm 185 ) [41].

There are just a few studies assessing MRU in patients with CHC. In a retrospective and multicentre study, Thongsawat et al. [42] evaluated MRU and direct medical costs of patients with $\mathrm{CHC}$ in Thailand. Though the most prevalent genotype in the Thai population is genotype 3, their results aligned with our findings - that is, less severe patients were less often admitted to hospitals and tended to have a shorter stay in the hospital than more severe patients did. Bonaventura et al. [43] reported a greater number of physician visits for patients with $\mathrm{HCV}$ infection treated with PegIFN/R than for those who were not treated. In a retrospective study, Nevens et al. [44] assessed MRU and costs in patients who were referred to a medical specialist to consider antiviral treatment and/or treatment for a complication of their disease. They reported more resource consumption and higher management costs in patients with advanced liver disease than in those with mild to moderate liver disease. Hospitalization rate further increased with more severe disease stage. Overall, our findings aligned 
with the results of these studies, though cross-comparison is not straightforward, given the differences in study design, patient characteristics and prior response status to PegIFN/R, treatment options, healthcare provision and cost structures.

\subsection{Study Strengths and Limitations}

Although these analyses made adjustments for baseline, clinical and treatment characteristics in healthcare resource utilization and total visit costs, there may be other factors that have an impact on these outcomes that were not captured in the clinical trials that formed the basis for this analysis. In addition, the trial sample size was determined in order to show clinical benefit, not significance in cost savings. As a result, the results presented here are from post hoc analysis that were not in the study protocol or the statistical analysis plan.

In addition, this study does not capture all of the direct costs, or any indirect costs, of treating HCV infections. The direct treatment costs for hepatitis $\mathrm{C}$ include not only the cost of providing outpatient care and hospitalization but also medication costs and laboratory tests. In addition, indirect costs could include benefits in terms of work productivity and presenteeism. Hence, the real non-drug savings may be higher than those presented here.

The results of the present study have strong implications for clinicians and policy makers, especially with the market authorization of new direct-acting antivirals (DAAs) and their combinations from late 2014 and beyond. It is anticipated that interferon-containing regimens will be replaced in the future by interferon-free regimens, which show cure rates higher than $90 \%$ and more tolerable side effects [33]. However, genotype 1 will still likely derive benefit from interferon-containing regimens, while keeping drug expenses manageable, especially in countries where healthcare budget would be limited. It could, therefore, also be of interest to acknowledge alternative approaches to generate additional savings and optimize budget planning. Our results support the implementation of a weight management program when caring for patients with $\mathrm{CHC}$. Additionally, given that the differences between the new DAAs will reside in their ease of usage, shortened treatment duration, costs and the high barrier to $\mathrm{HCV}$ resistance, policy makers should encourage early access to treatment, to limit the significant costs associated with advanced liver fibrosis. Finally, clinicians should take various patient characteristics into consideration to determine which drug regimen to use. In particular, treatment regimens should be used that reduce the occurrence of adverse events and that can shorten the treatment duration without affecting the likelihood of achieving SVR.

\section{Conclusions}

The MRU data collected in the SMV clinical development program indicate that SMV/PegIFN/R has lower non-drug MRU-related costs and an MRU pattern similar to that observed with PegIFN/R. This is in line with the reported efficacy and safety of SMV triple therapy, which was found to be an effective treatment for HCV infections, with $>80 \%$ of patients eligible for a shortened total treatment duration, and an incidence of anaemia, pruritus and rash similar to that observed with PegIFN/R alone.

The present study demonstrates that savings could be achieved when a treatment regimen is used that reduces the occurrence of rash and can shorten the treatment duration for a larger proportion of patients. Using the regression analyses presented here, it is possible to estimate the cost impact for specific patient populations with $\mathrm{HCV}$ on the basis of their demographics and clinical characteristics and the attributes of the selected drug regimens [41]. The study suggests that there are potential patient management and cost-effectiveness implications associated with the attributes of the antiviral treatments that are utilized and the populations to which they are applied. Further research comparing MRU and related costs between new DAAs would provide clinicians and payers with additional information for better resource allocation, treatment selection and budgetary planning.

Acknowledgments The authors acknowledge Chris Corbett and George Wan for their advice on the manuscript.

Conflict of interest The authors E.I.H.A. and J.K. declare no conflict of interest. K.C. is an employee of Janssen Pharmaceutica. This study was funded in full by Janssen Pharmaceutica, which holds the market authorization for simeprevir. All of the analyses were performed by E.I.H.A. The manuscript was written by E.I.H.A. and J.K. K.C. provided access to the simeprevir trial data and comments on the manuscript. The study guarantor is K.C. All authors have approved the final version of the article.

Open Access This article is distributed under the terms of the Creative Commons Attribution Noncommercial License which permits any noncommercial use, distribution, and reproduction in any medium, provided the original author(s) and the source are credited.

\section{References}

1. Hatzakis A, Wait S, Bruix J, Buti M, Carballo M, Cavaleri M, et al. The state of hepatitis B and C in Europe: report from the Hepatitis B and C Summit Conference. J Viral Hepat. 2011;18(Suppl 1):1-16.

2. Costella A, Goldberg D, Harris H, Hutchinson S, Lyons M, McCartney M, et al. Hepatitis C in the UK: 2013 report. London: Public Health England; 2013. 
3. Sánchez Á. Module XIX cost efficacy and cost-benefit of treatment of hepatitis C. Ann Hepatol. 2006;5(Suppl 1):S69-73.

4. Patruni B, Nolte E. Hepatitis C: A projection of the healthcare and economic burden in the UK. Santa Monica: Rand Corporation; 2013.

5. Backx M, Lewszuk A, White JR, Cole J, Sreedharan A, van Sanden S, et al. The cost of treatment failure: resource use and costs incurred by hepatitis $\mathrm{C}$ virus genotype 1-infected patients who do or do not achieve sustained virological response to therapy. J Viral Hepat. 2014;21(3):208-15.

6. Maasoumy B, Port K, Markova AA, Serrano BC, Rogalska-Taranta $\mathrm{M}$, Sollik L, et al. Eligibility and safety of triple therapy for hepatitis $\mathrm{C}$ : lessons learned from the first experience in a real world setting. PLoS One. 2013;8(2):e55285.

7. US Food and Drug Administration. Olysio (simeprevir) for the treatment of chronic hepatitis $\mathrm{C}$ in combination antiviral treatment. US Food and Drug Administration. 2013. http://www.fda. gov/forconsumers/byaudience/forpatientadvocates/ucm 377234 . htm. Accessed 20 Feb 2014.

8. Manns M, Marcellin P, Poordad F, de Araujo ES, Buti M, Horsmans Y, et al. Simeprevir with pegylated interferon alfa $2 \mathrm{a}$ or $2 b$ plus ribavirin in treatment-naive patients with chronic hepatitis $\mathrm{C}$ virus genotype 1 infection (QUEST-2): a randomised, double-blind, placebo-controlled phase 3 trial. Lancet. 2014;384(9941):414-26.

9. Jacobson IM, Dore GJ, Foster GR, Fried MW, Radu M, Rafalsky $\mathrm{VV}$, et al. Simeprevir with pegylated interferon alfa $2 \mathrm{a}$ plus ribavirin in treatment-naive patients with chronic hepatitis $\mathrm{C}$ virus genotype 1 infection (QUEST-1): a phase 3, randomised, double-blind, placebo-controlled trial. Lancet. 2014;384(9941): 403-13.

10. Department of Health. Reference costs 2011-2012. London: National Health Service; 2012.

11. Curtis L, Netten A. Unit costs of health \& social care 2008. Canterbury: University of Kent; 2008.

12. Curtis L. Unit costs of health \& social care 2007. Canterbury: University of Kent; 2007.

13. Office for National Statistics. Consumer price inflation reference tables, March 2013. UK Statistics Authority. 2013. http:// www.ons.gov.uk/ons/rel/cpi/consumer-price-indices/march-2013/ consumer-price-inflation-reference-tables.xls. Accessed 16 May 2013.

14. Liu L, Strawderman RL, Cowen ME, Shih YC. A flexible twopart random effects model for correlated medical costs. J Health Econ. 2010;29(1):110-23.

15. Romero-Gomez M, Berenguer M, Molina E, Calleja JL. Management of anemia induced by triple therapy in patients with chronic hepatitis $\mathrm{C}$ : challenges, opportunities and recommendations. J Hepatol. 2013;59(6):1323-30.

16. Jacobson IM, Kowdley KV, Kwo PY. Anemia management in the era of triple combination therapy for chronic HCV. Gastroenterol Hepatol (NY). 2012;8(9 Suppl 6):1-16.

17. Singh G, Issa D, Sedki E, Hanouneh I, Lopez R, Zein N, et al. Anemia in patients with chronic hepatitis $\mathrm{C}$ infection during triple therapy with telaprevir or boceprevir-a controlled study. J Antivir Antiretrovir. 2013;5:173-7.

18. Kistler KD, Molleston J, Unalp A, Abrams SH, Behling C, Schwimmer JB. Symptoms and quality of life in obese children and adolescents with non-alcoholic fatty liver disease. Aliment Pharmacol Ther. 2010;31(3):396-406.

19. Jensen DM, Pol S. IL28B genetic polymorphism testing in the era of direct acting antivirals therapy for chronic hepatitis $\mathrm{C}$ : ten years too late? Liver Int. 2012;32(Suppl 1):74-8.

20. Thompson AJ, Muir AJ, Sulkowski MS, Ge D, Fellay J, Shianna $\mathrm{KV}$, et al. Interleukin-28B polymorphism improves viral kinetics and is the strongest pretreatment predictor of sustained virologic response in genotype 1 hepatitis $\mathrm{C}$ virus. Gastroenterology. 2010;139(1):120-9.

21. Jacobson IM, McHutchison JG, Dusheiko G, Di Bisceglie AM, Reddy KR, Bzowej NH, et al. Telaprevir for previously untreated chronic hepatitis $\mathrm{C}$ virus infection. $\mathrm{N}$ Engl $\mathrm{J}$ Med. 2011;364(25):2405-16.

22. Lavanchy D. The global burden of hepatitis C. Liver Int. 2009;29(Suppl 1):74-81.

23. Lavanchy D. Evolving epidemiology of hepatitis C virus. Clin Microbiol Infect. 2011;17(2):107-15.

24. Hourigan LF, Macdonald GA, Purdie D, Whitehall VH, Shorthouse $\mathrm{C}$, Clouston A, et al. Fibrosis in chronic hepatitis C correlates significantly with body mass index and steatosis. Hepatology. 1999;29(4):1215-9.

25. Bressler BL, Guindi M, Tomlinson G, Heathcote J. High body mass index is an independent risk factor for nonresponse to antiviral treatment in chronic hepatitis C. Hepatology. 2003;38(3):639-44.

26. Hickman IJ, Powell EE, Prins JB, Clouston AD, Ash S, Purdie $\mathrm{DM}$, et al. In overweight patients with chronic hepatitis C, circulating insulin is associated with hepatic fibrosis: implications for therapy. J Hepatol. 2003;39(6):1042-8.

27. Colombo M, Fernandez I, Abdurakhmanov D, Ferreira PA, Strasser SI, Urbanek P, et al. Safety and on-treatment efficacy of telaprevir: the early access programme for patients with advanced hepatitis C. Gut. 2014;63(7):1150-8.

28. Dan AA, Martin LM, Crone C, Ong JP, Farmer DW, Wise T, et al. Depression, anemia and health-related quality of life in chronic hepatitis C. J Hepatol. 2006;44(3):491-8.

29. Bichoupan K, Schwartz JM, Martel-Laferriere V, Giannattasio ER, Marfo K, Odin JA, et al. Effect of fibrosis on adverse events in patients with hepatitis $\mathrm{C}$ treated with telaprevir. Aliment Pharmacol Ther. 2014;39(2):209-16.

30. Aapro MS, Cella D, Zagari M. Age, anemia, and fatigue. Semin Oncol. 2002;29(3 Suppl 8):55-9.

31. Sulkowski MS, Shiffman ML, Afdhal NH, Reddy KR, McCone J, Lee WM, et al. Hepatitis $C$ virus treatment-related anemia is associated with higher sustained virologic response rate. Gastroenterology. 2010;139(5):1602-11.

32. Poordad F. Big changes are coming in hepatitis C. Curr Gastroenterol Rep. 2011;13(1):72-7.

33. Pawlotsky JM. New hepatitis $C$ therapies: the toolbox, strategies, and challenges. Gastroenterology. 2014;146(5):1176-92.

34. Blatt C, da Cunha Bernardo N, Rosa J, Bagatini F, Alexandre R, Neto $G$, et al. An estimate of the cost of hepatitis $C$ treatment for the Brazilian health system. Value Health Reg Issues. 2012;1:129-35.

35. Stahmeyer JT, Rossol S, Bert F, Abdelfattah AM, Mauss S, Heyne R, et al. Epidemiology, treatment outcomes and costs of treating hepatitis $\mathrm{C}$ in routine care-results from a large multicenter trial. Value Health. 2013;16(7):A341-2.

36. Kenney JT Jr. Payers' management of oncology drugs: opportunities and challenges. Am Health Drug Benefits. 2014;7(3):123-4.

37. Lieberman SM, Lee J, Anderson T, Crippen DL. Reducing the growth of Medicare spending: geographic versus patient-based strategies. Health Aff (Millwood). 2003; Suppl Web Exclusives:W3-13.

38. World Health Organization. Towards universal access: scaling up priority HIV/AIDS interventions in the health sector: progress report 2010. Geneva: World Health Organization; 2010.

39. Siebert U, Wasem J, Rossol S, Sroczynski G, Aidelsburger P, Ravens-Sieberer $U$, et al. Antiviral treatment initiation costs in chronic hepatitis C. Gut. 2005;54(1):172-3.

40. Athanasakis K, Arzoumanidou D, Petrakis I, Karampli E, Theodoropoulou T, Retsa MP, et al. A cost-of-illness analysis of hepatitis C in Greece. Value Health. 2013;16(7):A496. 
41. Davis KL, Mitra D, Leteneux C, Bapat B, Naujoks C. PGI15 medical service utilization and costs by disease severity, sustained viral response, and genotype in European patients with chronic hepatitis C virus. Value Health. 2010;13(3):A71.

42. Thongsawat S, Piratvisuth T, Pramoolsinsap C, Chutaputti A, Tanwandee T, Thongsuk D. Resource utilization and direct medical costs of chronic hepatitis $\mathrm{C}$ in Thailand: a heavy but manageable economic burden. Value Health Reg Issues. 2014;3c:12-8.
43. Bonaventura M, Yuan Y, L'Italien G, Lescrauwaet B. The effect of hepatitis $\mathrm{C}$ treatment on health-related quality of life, work productivity, and healthcare resource use among patients in Europe. J Hepatol. 2012;56:S389-548.

44. Nevens F, Colle I, Michielsen P, Robaeys G, Moreno C, Caekelbergh K, et al. Resource use and cost of hepatitis C-related care. Eur J Gastroenterol Hepatol. 2012;24(10):1191-8. 